\title{
CLOUD COMPUTING: ENTERPRISE 2.0
}

\author{
James Lee, Seattle University, leej@seattleu.edu \\ Ben Kim, Seattle University,bkim@seattleu.edu \\ Peter Raven, Seattle University,pvraven@seattleu.edu
}

\begin{abstract}
Cloud computing is rapidly becoming the next generation of computing architecture. In the coming years, it will simplify corporate IT (information technology) operations, conceivably lowering the costs of IT maintenance. Cloud computing also enhances IT infrastructure by providing security, compliance, availability, and accessibility. Currently, vendors of cloud computing emphasize scalability and flexibility. One of the most important benefits of cloud computing is its capability of dealing with the fluctuating demands of IT. With service oriented architecture (SOA), software, and computing platforms, the IT infrastructure can be analogous to a power utility as consumers pay only as much as they use. Enterprise 2.0 is a new concept for building a corporate computing infrastructure using cloud computing and Web 2.0 technologies. This paper aims to provide a universally applicable conceptual framework for the Enterprise 2.0 platform by exploring the strategic implications for businesses using the acronym SLATES (Search, Links, Authoring, Tags, Extensions, Signals) [10]. Business managers will find this framework to be helpful to reengineer their business processes and structure in a new enterprise computing environment.
\end{abstract}

Keywords: Cloud Computing, Web 2.0, Enterprise 2.0, Web Browser, SLATES (Search, Links, Authoring, Tags, Extensions, Signals), SaaS, PaaS, IaaS, Salesforce.com

\section{INTRODUCTION}

With the advancement in networking and telecommunication technologies, business computing architecture has evolved from centralized mainframes and dumb terminals in the 1950s to client/server computing in the 1990s. In the 2000s, the wide availability of the Internet allowed businesses to use web services independent of the heterogeneous computers and operating systems. In the $2010 \mathrm{~s}$, we are observing cloud computing as a main force in business IT [7]. Cloud computing virtualizes the computing infrastructure and software applications over the Internet [8]. Consequently, businesses are becoming able to use computing resources as their needs arise without worrying about over- or under-provisioning. Hybrid IT and cloud computing now play major roles for IT department [4].

Many businesses are migrating to cloud computing for various reasons, including the following: no need for software installation on your company's computers, no need for system upgrade, no need for inflexible software license management, no need for internal IT maintenance. This type of cloud computing is called, Software as a Service - SaaS. Salesforce.com is an industry leading vendor of CRM (customer relationship management). As customers of Salesforce.com demanded more flexible ways for implementing CRM, Salesforce.com has launched a platform service called Force.com where customers can develop their own web applications. This type of cloud computing is called Platform as a Service - PaaS.

The major platform delivering cloud computing to users is the web browser. Web browser technologies with advanced scripting and database systems function as an interface encapsulating the heterogeneous details of various devices such as smart phones, laptops, tablets, servers, or desktop computers. With this newer and integrated platform, the web browser, Web 2.0 has evolved into one of the most influential communication media cultivating and building social relationships. Companies find their websites as one of the most effective communication channels with their customers. In other words, Web 2.0 can be used for enterprises to reap the benefits of social relationships via web technologies. In this paper, we provide a conceptual framework for how enterprise resources can be effectively organized and managed using Web 2.0 technologies.

\section{NEW PLATFORM PARADIGM - WEB BROWSER}

Today's web browser market has several market leaders: IE (Internet Explorer) from Microsoft, Firefox from Mozilla, Chrome from Google, Safari from Apple, and so on. Even though there are some minor differences, these browsers practically work in the same way. At present, web pages are created in compliance with the rules of XHTML specified by the W3 Consortium [16]. 
There were two landmark innovations in the development of web standards; XML and scripting (programs executed on the web browser platform). XML (eXtensible Markup Language) is for specifying how data are described while HTML5 is the basic backbone of web pages for reading content. In other words, we use HTML5 (previously known as HTML and XHTML) to build web pages and XML to exchange data between web applications. Scripting, meanwhile, provides application processing capabilities to web pages by inserting programming codes into XHTML documents. As a result, web pages have become more than just for presenting data. Rather they act like web applications (web apps) that can run on either server-side or client-side computers. One of the most widely used web apps is a shopping cart. Its business implications include enabling the end-users to purchase what they want directly on the website.

Instead of compiling the programs into machine code, interpreters on the web page build machine codes on the fly using a platform specific interpreter. Examples of interpreters include JavaScript virtual machine, PHP processors, .NET framework, Flash player, etc. Interpreters are freely distributed based on each machine's platform such as operating systems or various web browsers. Companies develop websites to communicate with customers and business partners. A majority of dynamic web pages are built as web applications. This enables web browsers to be a new platform for interactive communications [9].

\section{WEB 2.0}

The use of the World Wide Web has expanded to areas where massive collaboration can occur, including social networking, blogging, Wikipedia, etc. The Web and database systems are an integral part of these new applications [9]. Using this technical environment, we can connect data, information, processes, knowledge, businesses, and people. This is known as Web 2.0, the haven of relationship management, where massive collaboration among businesses or people can be enabled [13]. Web 2.0 is considered as the second stage of development of the World Wide Web. It is characterized by the change from static web pages to dynamic or user-generated content and the growth of social networking.

One of the major impacts of Web 2.0 on communications is relationship management over the Web. With this social establishment over the Web (such as social networking, Wikipedia, blogging, etc.), companies can manage and flourish relationships among resources via Web 2.0, increasing and meeting opportunities from today's connectivity from communication convergence between channels and devices. $[6,12]$. These new types of businesses enabled by Web 2.0 technologies are called Enterprise 2.0.

IT has been providing strategic value through business intelligence through CRM (customer relationship management). CRM not only processes communications with customers, but also analyzes what customers want or how to effectively re-act and pro-act with customers. As CRM has been positioned as a corporate major business function, vendors started providing many different solutions. One of the easiest ways to adopt and operate CRM right away is the solution by cloud computing such as industry leading Salesforce.com. As the CRM domain moves to a cloud computing platform, it incorporates the features and benefits of Web 2.0.

The rest of this paper suggests more universally applicable practices for the Enterprise 2.0 platform. This framework offers the "business strategies" for the Enterprise 2.0 model, SLATES [10], which assists managers to operate organizations at the optimal level. Significant benefits of Enterprise 2.0 can be summarized into six categories: search, links, authorship, tags, extensions, signals (SLATES). The leading CRM provider, Salesforce.com, is analyzed within the SLATES framework. This will show how cloud computing today is successful and is becoming a dominant computing environment for enterprises.

\section{BUSINESS STRATEGIES OF ENTERPRISE 2.0 ELEMENTS}

Web 2.0 can provide the paradigm shift in many dimensions of organizational performance, such as collective intelligence, peer-production, lightweight programming models, and software above the level of a single device. The World Wide Web has become a major providing a global communication channel covering all regions of the earth. Also, it provides a hypertext structure where any files in any computers can be linked regardless of the location and time. Many successful websites host such a large-scale communications between people, such as Wikipedia, Yahoo, Google, eBay as well as social networking sites like Facebook. Through this massive collaboration among people, collective intelligence can be developed to solve many new business problems. In developing open source software, collective intelligence played an indispensable role enabling peer production and raising the productivity. 
Collective intelligence and open source culture on the web have changed the programming models. Due to the open nature of web sites, web developers are sharing the web applications programming resources. Programming frameworks, libraries, and plugins are fundamentally providing lightweight programming models, giving flexibility and portability on web applications.

The concept of software is changing. Unlike in the past, software is no longer limited to any particular type of operating systems such as Windows. As we discussed earlier, the Web is considered as a new platform and it has become the "Interface" of a standard implementation of cloud computing. Since the Web browser hides the details and heterogeneity of the underlying computing devices, software is developed independently of the particular computing platforms and delivered as a service as we can see in cloud computing (Software as a Service or SaaS).

\section{SLATES Framework}

McAfee [10] proposed the SLATES (Search, Links, Authoring, Tags, Extensions, Signals) Framework to describe Enterprise 2.0. In this paper, we use the SLATES Framework to explain how Enterprise 2.0 can be implemented in business.

Search The first component of SLATES is search. Search capability provides Enterprise 2.0 with reuse, leverage, and ROI (Return on Investment) of information. Web 2.0 runs information and communication technologies over the Internet, forming various work groups that expand to intranets and extranets. This includes the Internet, LAN (Local Area Networks) and WAN (Wide Area Networks) for business, email and online chat/bulletin boards and real-time video conferencing. These technologies allow smaller workgroups as part of a larger company to operate independently of each other, across a room or across the globe. Search also focuses on individual tasks from mass-customization functions from the current CRM where information can be tailored before presented to the users.

Link Companies now can direct web services using uniform resource identifiers (URIs) to forge thousands of interconnections anytime and anywhere. Because companies use cloud computing these days, integration between services can be very flexible (or tend to be temporal), but can be done instantly in both ways, vertically or horizontally. While each member of Enterprise 2.0 is indeed linked to a much larger corporate structure which oversees them all, they each remain a separate and often self-sustaining entity. They rely very little on outside help from the corporate headquarter and work independently of the majority of other members. Each member of the organization is charged with an explicit task to complete as it relates to the overall functions of the organization. Often times, after this single goal is completed, the link between the organization and the entity is dissolved until a further need for it is realized. At this point, the link is re-established.

Authorship Employee empowerment is becoming stronger in the Enterprise 2.0 platform. The ease with which two members of an Enterprise 2.0 can communicate allows them to pool their resources, even with members not directly involved in a specific project. It fundamentally provides a high level of modularity in collaborative culture. By its nature, Enterprise 2.0 fosters camaraderie between the members even in the absence of face-to-face communications. Since the built-in communications tools are so easy to access and use, relationships form between members who haven't even met. A corporate culture formed out of camaraderie produces a highly collaborative environment unlike traditional organizations where such extensive communication is not required.

Tags All resources are ready to be tagged, allowing natural, organic, on-the-fly organization of data from every point of view. Members of an Enterprise 2.0 collaborate to share their knowledge gained from individual activities performed. Since collaboration is facilitated through the communication channels that are afforded through the Enterprise 2.0, it is common to find "knowledge bases" or other database systems that contain information and documents pertaining to past experience. This allows the switching principle - a fundamental advantage that an Enterprise 2.0 has over traditional organizations. Because the links between organizational functions are largely electronic and non-physical, it is easy to replace a weak component with a stronger one. In other words, Enterprise 2.0s are often formed to fill temporary needs, only extending to the end of the specific project that is charged to them. As a continuation of flexibility, the Enterprise 2.0 is characterized by vague boundaries as to the extent of its use and purpose. Since small tweaks can easily affect the overall organization, it is quite possible to extend the boundaries of an organization so that they encompass new purpose, people or controls. 
Extensions All resources have been documented with self-organizing culture, extending knowledge by mining patterns and user activity, i.e., extensions. The nature of global diversity and the ability to locate organizational functions across the globe creates a diverse environment for the entire organization. Since the members are at different locations and charged with different tasks, diversity that is only found in the very largest multi-national corporations can be managed effectively. It also allows the transience of Enterprise 2.0 components to be regularly switched in and out of use, altering the overall structure of the organization. Because this can be done with relatively little impact, the frequency with which components are switched is high and allows each organization to quickly adapt itself to a changing environment. The lack of physical interaction places a higher regard on the trust that exists between each entity involved in the organization. Since fewer "checks and balances" can be placed on appropriate departments, management and other entities trust that they will complete the appropriate work on time or be straightforward about delays or problems. If two entities working on a project together separated by thousands of miles are unwilling to trust each other, the work slows and suffers to a critical point. This may lead to a new problem to be resolved.

Signals As one of the major drivers in Web 2.0, signals create a logical level of communications from the client/server architecture that mainly interconnects resources using URIs (Uniform Resource Identifiers). This makes information consumption more efficient by pushing out the changes. For example, customer based products can be accomplished through outsourcing the works to a separate organization or through the use of a virtually connected inter-organizational unit located closer to the customers. Each individual unit of an Enterprise 2.0 is expected to make a contribution towards a given goal.

\section{CLOUD COMPUTING IN BUSINESS PRACTICE}

Business practices have evolved as IT has advanced. One of the significant business valuing with modern IT had started from Michael Porter [14]. Business processes are given values by Porter's value chain [14]. One of the later valuing business practices emerged with customer-oriented services by Theodore Levitt and Peter Drucker [2]. As computing has evolved rapidly in last two decades, the field of IT also transformed into the Services Wave as predicted by Bill Gates [3]. The services wave was initiated by XML and web services, service-oriented architecture (SOA) is well accepted by companies, providing remote orchestration of application components. Computing as a service model provides multiple options for organizational IT structure. As a result, a traditional IT department is no longer tenable and cloud services have become less expensive [5].

Companies are attracted to the following opportunities of cloud computing: no need for software installation on your company's computers, no need for a system upgrade, no need for inflexible software license management, no need for internal IT maintenance. This trend is called, Software as a Service - SaaS [1]. Besides its scalability and superior IT architecture, there are many benefits of cloud computing, such as better individual performance, facilitating collaborations, mining insights, cost, reliability, security, regulation, etc. [11]

Salesforce.com is an industry leading vendor of customer relationship management (CRM) [15]. As customers of Salesforce.com demanded more flexibility in CRM implementation, Salesforce.com launched a platform service called Force.com where customers can develop their own web applications. This is called the Platform as a Service - PaaS. Another type of cloud computing is Infrastructure as a Service (IaaS). Using IaaS, companies do not need to maintain hardware, computing power, storage, operating systems or other infrastructure. Examples of IaaS are Amazon Elastic Compute Cloud, Amazon Simple Storage Service, Google Data Center, SunGard IaaS, and others.

\section{A CASE: SALESFORCE.COM}

Salesforce.com has been primarily a CRM solution provider in the Software as a Service (SaaS) space. Lately, it has also extended its territory to the Platform as a Service (PaaS) with Force.com. In this section, we investigate how SaaS and PaaS can be understood and evaluated in the framework of Enterprise 2.0. The data was collected by the authors with Salesforce.com developers. A primary investigator explained the SLATE to the developers at Salesforce.com and then they provided how the system could work in the SLATE framework.

\section{Search}

The Force.com platform stores data in a similar fashion to a relational database. Force.com platform users can create objects, a similar representation to a table in a relational database, and use these objects to store data in the cloud. The platform provides an ad-hoc search feature that allows users to search and display data in a 
user-friendly web user interface without the need for in-depth knowledge of SQL. In addition to the search, the platform provides analytics tools that allow users to create dashboards and reports and monitor real time data. With the ease of creating objects and managing data in the cloud, coupled with the search and analytics tools, users can concentrate in monitoring key performance indicators to steer the business to success.

\section{Links}

Each record stored in the Force.com object, by default, comes with a unique identifier. When combined with salesforce.com domain, the unique identifier acts as a uniform resource identifier (URI) to retrieve and view the data. The data is displayed with the platform's customizable web user interface and security setting. Since objects can form a parent-child relationship, platform users hyperlink to display the relationship between the parent and the child records, and vice versa.

\section{Authorship}

Salesforce Content is a Salesforce CRM feature that can be added into the Force.com platform. With Salesforce Content, platform users can upload documents in the cloud and share them with others. Other users can subscribe to documents and receive notifications when the author uploads a new document version. Salesforce Content also allows users to provide feedback on the uploaded documents, allowing rich interactions between the users and the authors.

\section{Tags}

The Force.com search \& analytics tools empower users with various ways to retrieve, organize and present data in a structured manner. In addition to the search \& analytics tools, the platform provides its users with a free-form tagging mechanism, as a way to categorize and bookmark data. Tags can be set as private or public. If set to public, other users holding a platform account can view the public tags and the records associated with each tag. They can also contribute to these public tags by tagging additional records with identical keywords.

\section{Extensions}

With the latest acquisition of Radian6, salesforce.com is adding social media monitoring tools into the platform monitoring tool. Radian6 provides a unique technology to monitor and measure conversation in social networking sites, such as Facebook, Twitter, and blogs. Once integrated with the Force.com platform, this tool will allow the platform user to extend knowledge about peoples' sentiments of brands or specific business activities by mining the patterns and the user activities from social networking sites.

\section{Signals}

Salesforce Chatter, a Facebook-like feature, is an enterprise collaboration feature that can be enabled in the Force.com platform. Chatter allows users to follow people, documents or records in the platform. The Chatter API (application programming interface) posts changes related to items that users follow in their Chatter profile page, allowing the user to receive notifications about the changes.

In addition to Salesforce Chatter, the platform provides a point-and-click workflow builder and an Apex programming language. With workflows and Apex programming language, platform users can develop custom business logics that allow the system to send an email notification or assign a task to specific platform users. Users who are assigned the task will receive an alert in a small pop-up window when the user logs into the system.

\section{CONCLUSIONS}

This paper is an effort to conceptualize the new era of cloud computing in the business field. As an example, Enterprise 2.0 [6] with cloud computing solutions is identified. With SaaS and PaaS, Salesforce.com meets high expectations for business organizations. With business strategies provided in this study, companies can find guidelines for building Enterprise 2.0 with cloud computing. As with Web 2.0, Enterprise 2.0 will facilitate the communications and relationships among employees, and it will extend a reach with customers and business partners. Also, the data collected in the process can be analyzed for raising the level of business intelligence using big data technologies and data mining.

The SLATE framework provides an excellent perspective for how relationships can be organized. However, it lacks an explicit example or scenarios. This paper shows how current cloud computing technology can be adopted to implement the SLATE framework. Furthermore, as a specific example, we explained the SLATE framework using Salesforce.com to help the readers to understand the benefits of Web 2.0 and Enterprise 2.0. . 


\section{REFERENCES}

1. Armbrust, M., Fox, A., Griffith, R., Joseph, A. D., Katz, R., Konwinski, A., Lee, G., Patterson, D., Rabkin, A., Stoica, I. \& Zaharia, M. (2010). "A View of Cloud Computing," Communications of the ACM, Vol. 53, No. 4 , pp. 50-58.

2. Chesbrough, H. (2011). "Bringing Open Innovation to Services," MIT Sloan Management Review, Vol. 52, No. 2, pp. 84-90.

3. CNET. (2005). "Gates memo: Brace for 'services wave'," November 9, 2005, http://news.cnet.com/2100-1016_3-5942191.html

4. Gartner, Inc. (2012). "Gartner Identifies the Top 10 Strategic Technology Trends for 2013," http://www.gartner.com/newsroom/id/2209615.

5. Golden, B. (2013). "How Cloud Computing Changes Enterprise IT Economics," CIO, http://www.cio.com/article/print/733072.

6. Hinchcliffe, D. (2007). "The state of Enterprise 2.0," ZDNet, http://www.zdnet.com/blog/hinchcliffe/the-state-of-enterprise-2-0/143.

7. IBM Smart Business. (2010). "Dispelling the vapor around cloud computing," Thought Leadership White Paper, ftp://public.dhe.ibm.com/common/ssi/ecm/en/ciw03062usen/CIW03062USEN.PDF. This reference is not cited

8. Kouyoumjian, V. (2010). The New Age of Cloud Computing and GIS, ArchWatch: esri, http://www.esri.com/news/arcwatch/0110/feature.html.

9. Lai, L. S. L. \& Turban, E. (2008). "Groups Formation and Operations in the Web 2.0 Environment and Social Networks," Group Decis Negot, Vol. 17, pp. 387-402.

10. McAfee, A. P. (2006). "Enterprise 2.0: The Dawn of Emergent Collaboration," MIT Sloan Management Review, Vol. 47, No. 3, pp. 21-28.

11. McAfee, A. P. (2011). "What Every CEO Needs to Know About The Cloud," Harvard Business Review, November, 2011.

12. Miller, R. (2007). "Enterprise 2.0 Definition and Solutions," CIO, http://www.cio.com/article/print/123550.

13. O'Reilly, T. (2005). "What is Web 2.0," O'Reilly, http://oreilly.com/lpt/a/6228.

14. Porter, M. (1998). Competitive Advantage: Creating and Sustaining Superior Performance, Free Press, $1^{\text {st }}$ ed.

15. Salesforce.com (2012), "Software as a Service," http://www.salesforce.com/saas/.

16. World Wide Web Consortium, http://www.w3.org/ 